\title{
Cat Scratching Your Valve: An Elusive Case of Bartonella Endocarditis
}

\author{
Ritu Nahar, MD and Evan Caruso, MD
}

\section{INTRODUCTION}

Bartonella Henselae is an uncommon, but significant cause of "culture-negative" endocarditis. While six Bartonella species have reportedly caused infective endocarditis (IE) in humans, the vast majority of cases are secondary to either $B$. quintana or B. henselae. The epidemiologic features of patients predisposed to Bartonella endocarditis are varied. Alcoholism, body lice infestation, and homelessness have been associated with $B$. quintana endocarditis, while $B$. henselae endocarditis has been linked to prior valvular disease and cat exposure. ${ }^{1-4}$ Patients with Bartonella endocarditis have clinical manifestations similar to those seen with traditional forms of subacute bacterial endocarditis. However, the rarity of the disease compounded by limitations of diagnostic testing make this entity a diagnostic challenge. This case exemplifies a classic presentation of Bartonella endocarditis while highlighting the systemic repercussions of inadequate source control and challenges associated with surgical intervention.

\section{CASE PRESENTATION}

A 53-year-old female with a history of dysautonomia status post pacemaker over 10 years ago, membranoproliferative glomerulonephritis (MPGN), and chronic pulmonary embolism (not on anticoagulation) presented to an outside hospital with two years of fatigue, weakness, and weight loss complicated by unexplained fevers for 2 weeks. The patient was initially treated with broad spectrum antibiotics, but fevers persisted. Infectious work-up at outside hospital was negative. Upon delving further into her social history, the patient endorsed extensive contact with cats, assisting in kitten birthing 6 months prior to presentation. Physical exam revealed a 2/6 systolic murmur at the left lower sternal border that was louder with inspiration. On transthoracic echocardiography, a $2.2 \times 1.9$ echodensity was visualized adjacent to the tricuspid valve in close proximity to the right ventricular (RV) pacemaker lead with smaller echodensities on the atrial side of the RV pacemaker lead. Broad-spectrum antibiotics were initiated but the patient continued to spike fevers as high as $104^{\circ} \mathrm{F}$. Blood cultures remained sterile despite prolonged incubation periods. Transesophageal echocardiography 5 days later demonstrated progression of lesions, now revealing a $3.9 \times 2.5 \mathrm{~cm}$ mass stemming from the RV pacemaker lead. On the 5th hospital day, the patient developed severe pleuritic chest pain. Computed tomography (CT) of the chest demonstrated wedge shaped peripheral opacities suspicious for lung infarct from septic emboli versus thromboemboli.

\section{DIFFERENTIAL DIAGNOSIS}

Patients with valvular/pacemaker vegetations in the absence of overt bacteremia typically result from marantic endocarditis secondary to a noninfectious etiology or culture-negative endocarditis from an infectious source. ${ }^{5}$ Marantic vegetations (nonbacterial thrombotic endocarditis) refers to a spectrum of lesions generally seen in the setting of hypercoagulable states, malignancy, and rheumatologic conditions including systemic lupus erythematosus (Libman-Sacks endocarditis). Interestingly, our patient had a history of pulmonary embolism diagnosed the year prior in the setting of antithrombin deficiency which, compounded by her biopsy proven MPGN, raised concern for a possible thrombotic endocarditis or verrucous endocarditis

The leading causes for negative cultures in infective endocarditis include prior antimicrobial treatment as seen with our patient and insufficient microbiological techniques. While HACEK organisms (Haemophilus aphrophilus; Actinobacillus actinomycetemcomitans; Cardiobacterium hominis; Eikenella corrodens; and Kingella kingae) were conventionally believed to be culture-negative, they have since been more successfully cultured with the assistance of modern culture systems. ${ }^{6}$ Instead, the predominating causative agents of blood culture-negative endocarditis are fastidious organisms such as zoonotic agents and fungi. C. burnetii and Bartonella species are two such pathogens with prevalence based on exposure to epidemiological risk factors. Of note, T. whipplei may also be an underappreciated source of culture-negative IE with Mycobacteria being a rarer cause.?

Our patient underwent a thorough rheumatologic and infectious work-up. Her extensive kitten exposure combined with iatrogenic interruption of her valve (pacemaker placement) significantly increased the risk for a possible Bartonella infection. Diagnostic tests for culture-negative endocarditis include unique culturing 
techniques, molecular tests including polymerase chain reaction (PCR) and serologic assays, and lastly direct histopathology staining of valvular tissue. She was found to have positive Bartonella serology with an elevated Bartonella henselae immunoglobulin G (lgG) level at 1:512. Interestingly, her B. Quintana IgG was also 1:512 likely secondary to cross-reactivity, which is common between IgG among Bartonella species. Of note, the recommended cutoff for definitive positivity in patients with suspected Bartonella endocarditis is a titer of $\geq 1: 800$ for IgG antibodies to either Bartonella henselae or Bartonella quintana. ${ }^{8}$ Diagnosis was eventually confirmed by positive blood PCR for Bartonella.

\section{OUTCOME AND FOLLOW-UP}

The patient was started on doxycycline (given continued symptomatic fevers) and deemed appropriate for pacemaker and lead removal. The decision was also made to initiate anticoagulation given evolving lung infarcts on CT. Management was complicated by heparin resistance requiring antithrombin concentrate pre- and intra-operatively. On hospital day 10, the patient underwent successful cardiopulmonary bypass surgery for pacemaker and associated mass removal. Of note, the patient was not a candidate for percutaneous lead extraction due to the size of her vegetation; thus, earlier detection may have precluded necessitating surgical intervention. Post-operatively she was initiated on a 6-week course of doxycycline, rifampin, and ceftriaxone. Gentamycin was avoided given her renal impairment from MPGN. Upon confirmation of Bartonella blood PCR, ceftriaxone was discontinued and the patient completed a 6-week course of oral doxycycline and rifampin.

\section{DISCUSSION}

This case highlights the importance of eliciting epidemiologic risk factors when working up fevers of unknown origin. Systemic complications our patient endured from lack of prompt intervention include recurrent hospitalizations and septic lung emboli. Additionally, Bartonella endocarditis is associated with development of immune-complex glomerulonephritis. ${ }^{9}$ Thus her MPGN may also in fact be a manifestation of her untreated endocarditis. Blood cultures for Bartonella henselae are seldom positive, potentially a result of intraerythrocytic sequestration of the pathogen. Therefore, specialized culturing, molecular assays, and histopathological evaluation of valvular tissue are generally required to establish the diagnosis.

Medical management for Bartonella endocarditis is comprised of dual therapy with doxycycline and gentamicin. Gentamicin may be replaced by rifampin if the patient has renal impairment. Ceftriaxone can be added for additional coverage of culture-negative endocarditis if definitive diagnosis of Bartonella has not been established. ${ }^{10}$ Of note, our patient had actively evolving lung infarction from septic emboli and recurrent symptomatic fevers resulting in hemodynamic instability, thus surgical removal of the pacemaker and leads was indicated. While endocarditis is an established predisposing factor for decreased heparin responsiveness during cardiopulmonary bypass, the mechanism of this phenomena and how this alters management pre and intra-operatively is an area of research requiring further evaluation. ${ }^{11}$

\section{KEY POINTS}

Recognizing and appropriately diagnosing Bartonella endocarditis is critical in preventing systemic complications via earlier surgical intervention for source control augmented with adequate antibiotic coverage.

\section{REFERENCES}

1. Raoult D, Fournier PE, Drancourt M, et al. Diagnosis of 22 new cases of Bartonella endocarditis. Ann Intern Med 1996;125:646-652.

2. Spach DH, Kanter AS, Daniels NA, et al. Bartonella (Rochalimaea) species as a cause of apparent "culture-negative" endocarditis. Clin Infect Dis 1995; 20:1044-1047.

3. Raoult D, Fournier PE, Vandenesch F, et al. Outcome and treatment of Bartonella endocarditis. Arch Intern Med 2003;163:226-230.

4. Fournier PE, Lelievre H, Eykyn SJ, et al. Epidemiologic and clinical characteristics of Bartonella quintana and Bartonella henselae endocarditis: a study of 48 patients. Medicine (Baltimore) 2001; 80:245-251

5. Fournier $P E$, Thuny $F$, Richet $H$, et al. Comprehensive diagnostic strategy for blood culture-negative endocarditis: a prospective study of 819 new cases. Clin Infect Dis 2010; 51:131-140

6. Petti CA, Bhally HS, Weinstein MP, et al. Utility of extended blood culture incubation for isolation of Haemophilus, Actinobacillus, Cardiobacterium, Eikenella, and Kingella organisms: a retrospective multicenter evaluation. J Clin Microbiol 2006; 44:257-259.

7. Fournier PE, Thuny F, Richet H, et al. Comprehensive diagnostic strategy for blood culture-negative endocarditis: a prospective study of 819 new cases. Clin Infect Dis 2010; 51:131-140

8. Raoult D, Casalta JP, Richet H, et al. Contribution of systematic serological testing in diagnosis of infective endocarditis. J Clin Microbiol 2005: 43:5238-5242.

9. Van Haare Heijmeijer S, Wilmes D, Aydin S, et al. Necrotizing ANCA-Positive Glomerulonephritis Secondary to Culture-Negative Endocarditis. Case Rep Nephrol 2015; 2015:649763.

10. Baddour LM, Wilson WR, Bayer AS, et al. Infective endocarditis: diagnosis, antimicrobial therapy, and management of complications: a statement for healthcare professionals from the Committee on Rheumatic Fever, Endocarditis, and Kawasaki Disease, Council on Cardiovascular Disease in the Young, and the Councils on Clinical Cardiology, Stroke, and Cardiovascular Surgery and Anesthesia, American Heart Association: endorsed by the Infectious Diseases Society of America. Circulation 2005;111:e394-434.

11. Na S, Shim JK, Chun DH, Kim DH, Hong SW, Kwak YL. Stabilized infective endocarditis and altered heparin responsiveness during cardiopulmonary bypass. World J Surg. 2009:33:1862-1867. 